\title{
Outcomes Following Implantation of Two Second- Generation Trabecular Micro-Bypass Stents in Patients with Open-Angle Glaucoma on One Medication: 18-Month Follow-Up
}

Richard Lindstrom - Richard Lewis · Dana M. Hornbeak · Lilit Voskanyan •

Jane Ellen Giamporcaro · John Hovanesian · Steven Sarkisian

Received: August 17, 2016 / Published online: October 13, 2016

(C) The Author(s) 2016. This article is published with open access at Springerlink.com

\begin{abstract}
Introduction: The study objective was to evaluate the intraocular pressure (IOP) and medication-lowering effect of 2 second-generation trabecular micro-bypass
\end{abstract}

Enhanced content To view enhanced content for this article go to http://www.medengine.com/Redeem/ 0D07F0600A84C640.

Electronic supplementary material The online version of this article (doi:10.1007/s12325-016-0420-8) contains supplementary material, which is available to authorized users.

R. Lindstrom $(\bowtie)$

Minnesota Eye Consultants, Minneapolis, MN, USA

e-mail: rllindstrom@mneye.com

R. Lewis

Sacramento Eye Consultants, Sacramento, CA, USA

D. M. Hornbeak · J. E. Giamporcaro

Glaukos Corporation, San Clemente, CA, USA

L. Voskanyan

S.V. Malayan Ophthalmology Centre, Yerevan, Armenia

J. Hovanesian

Harvard Eye Associates, Laguna Hills, CA, USA

S. Sarkisian

Dean A. McGee Eye Institute, University of

Oklahoma Health Science Center, Oklahoma City,

OK, USA stents in eyes with open-angle glaucoma (OAG) on one preoperative medication.

Methods: Fifty-seven qualified phakic eyes with OAG on 1 medication, preoperative medicated IOP of $18-30 \mathrm{mmHg}$, and preoperative unmedicated (post-washout) IOP of 22-38 mmHg underwent implantation of 2 second-generation trabecular micro-bypass stents in a standalone procedure. Evaluations included IOP, best-corrected visual acuity, medication use, fundus and slit lamp examinations, visual field, cup to disc ratio, pachymetry, and complications and interventions. Subjects have been followed for 18 months, and follow-up is ongoing.

Results: At Month 12 postoperative, 100\% of eyes had achieved an IOP reduction $\geq 20 \%$ (100\% had IOP $\leq 18 \mathrm{mmHg}$ and $67 \%$ had IOP $\leq 15 \mathrm{mmHg}$ ) without medication versus preoperative unmedicated IOP, and 75\% had IOP reduction $\geq 20 \%$ without medication versus preoperative medicated IOP. The Month 12 mean unmedicated IOP had decreased by $42 \%$, to $14.2 \pm 1.9 \mathrm{mmHg} \quad$ vs $\quad 24.4 \pm 1.3 \mathrm{mmHg}$ preoperatively, and this reduction was maintained through 18 months $\quad(14.4 \pm$ $2.1 \mathrm{mmHg}$ ). A high safety profile was observed. 
Conclusion: In this prospective, open-label, single-arm study, the standalone implantation of two second-generation trabecular micro-bypass stents in OAG patients on 1 preoperative medication resulted in IOP reduction to $\leq 15 \mathrm{mmHg}$ and elimination of medication through 18 months, with favorable safety.

Trial Registration: ClinicalTrials.gov identifier, NCT02868190.

Funding: Glaukos Corporation, San Clemente, CA.

Keywords: Glaucoma; IOP; Medication; MIGS; Ophthalmology; Trabecular micro-bypass

\section{INTRODUCTION}

The most important risk factor for glaucoma, the leading cause of permanent blindness worldwide [1], is elevated intraocular pressure (IOP). Thus, a central goal of all glaucoma therapy is IOP reduction, which can be achieved through pharmacologic, laser, or surgical means. Traditionally, trabeculectomy has been considered the gold standard of glaucoma surgery, but typically it has been reserved for more advanced stages of the disease [2]. Over the past decade, the development of microinvasive glaucoma surgery (MIGS) procedures has increased the surgical options available to clinicians treating mild to moderate open-angle glaucoma (OAG). The first FDA-approved MIGS device, the first-generation iStent (iStent $^{\circledR} \quad$ Trabecular Micro-Bypass, Glaukos Corporation, San Clemente, CA, USA), is a single-piece titanium stent designed to bypass the main site of aqueous fluid outflow resistance in OAG, the trabecular meshwork, with minimal trauma to intraocular and extraocular structures. A substantial body of literature has shown that $a b$ interno implantation of these stents can reduce IOP and medication burden for up to 5 years postoperatively in patients with mild to moderate OAG. Key studies have evaluated outcomes after implantation of single [3-6] and multiple [7] iStent devices during cataract surgery, and with multiple iStent devices as a standalone procedure [8-11].

More recent years have brought the advent of second-generation trabecular micro-bypass stents (iStent inject $^{\circledR}$ Trabecular Micro-Bypass, Glaukos Corporation, San Clemente, CA, USA). Several studies have demonstrated substantial IOP and medication reduction following implantation of 2 iStent inject devices during cataract surgery [12] or in standalone procedures [13-16]. In addition to their clinical effectiveness, these devices have shown a favorable safety profile, which is an important advantage over traditional surgeries such as trabeculectomy or tube placement, or more recently developed suprachoroidal stents $[17,18]$.

In the present study, we tested the hypothesis that IOP and medication burden would be reduced following the standalone implantation of 2 second-generation iStent inject devices in eyes with OAG not controlled on 1 preoperative medication, while maintaining favorable safety parameters. This report covers outcomes in 57 eyes through 18 months postoperation, and follow-up is continuing through 5 years.

\section{METHODS}

This was a prospective, unmasked, single-arm study designed to enroll phakic or pseudophakic (with posterior chamber intraocular lens) subjects with OAG, medicated 
IOP at screening of $18-30 \mathrm{mmHg}$ on 1 topical ocular hypotensive medication, and unmedicated (post-washout) IOP of 22-38 mmHg. Medication washout included 5 days for carbonic anhydrase inhibitors, 2 weeks for alpha agonists, and 4 weeks for beta blockers and prostaglandin analogues. Subjects were to have cup to disc (C:D) ratio of $\leq 0.9$, best-corrected visual acuity (BCVA) of $20 / 100$ or better, and normal angle anatomy. Exclusion criteria included pseudoexfoliative, pigmentary, uveitic, traumatic, neovascular, or angle-closure glaucoma; glaucoma associated with vascular disorders; and prior glaucoma surgery of any kind.

This single-site study was conducted at the S.V. Malayan Ophthalmological Center in Yerevan, Armenia. All surgeries were performed by the staff surgeon (L. Voskanyan) and 10 visiting surgeons from the MIGS Study Group (Supplementary Material-Appendix 1). Pre- and postoperative examinations were completed by glaucoma-trained ophthalmologists at the Center. All procedures followed were in accordance with the ethical standards of the responsible committee on human experimentation (institutional and national) and with the Helsinki Declaration of 1964, as revised in 2013. Informed consent was obtained from all patients for being included in the study. The ClinicalTrials.gov registration number for this study is NCT02868190.
As described previously in detail [12-15], the second-generation iStent inject device is a single-piece, titanium, heparin-coated stent with $360 \mu \mathrm{m}$ length, $230 \mu \mathrm{m}$ maximum width, and multiple lateral outlet lumens to facilitate aqueous fluid outflow (Fig. 1). Two stents are pre-loaded in a single disposable stainless steel inserter, which is advanced $a b$ internally through a temporal clear corneal incision to implant 2 stents through the trabecular meshwork into the nasal Schlemm's canal at approximately 2 clock-hours apart. The bypassing of the trabecular meshwork is designed to promote aqueous outflow and thereby decrease IOP. Using this technique, all subjects had implantation of 2 iStent inject devices in the nasal Schlemm's canal at approximately 2 clock-hours apart. Postoperatively, patients received topical anti-infective medication (for 1 week) and anti-inflammatory medication (for 4 weeks).

Efficacy and safety evaluations included IOP measurement (Goldmann applanation tonometry), BCVA (Early Treatment Diabetic Retinopathy Study scale), slit lamp biomicroscopy, indirect ophthalmoscopy, C:D ratio, visual field (Swedish Interactive Thresholding Algorithm Standard 24-2 automated perimetry), pachymetry, medication use, and ocular complications and secondary surgical interventions. Postoperative examinations occurred at Day 1; Week 1;

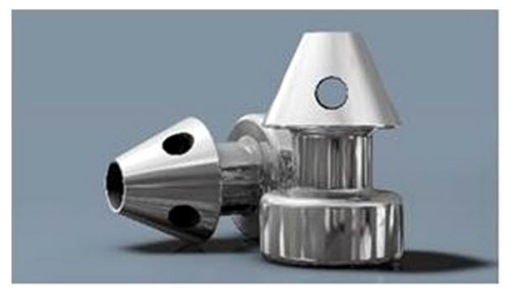

iStent inject stents

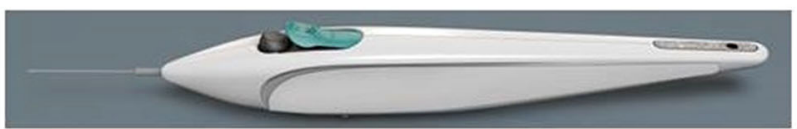

iStent inject Trabecular Micro-Bypass Stent System

Fig. 1 The iStent inject and iStent inject trabecular micro-bypass stent system 
Months 1, 3, 6, and 12; and are planned for every 6 months, thereafter until Month 60 . Additional ocular hypotensive medication was to be started if postoperative IOP exceeded $21 \mathrm{mmHg}$ at any postoperative visit, and/or in the case of concerning optic nerve findings, at the discretion of the investigator.

The primary efficacy endpoint was the proportion of patients with a Month 12 mean unmedicated IOP reduced by $\geq 20 \%$ versus baseline unmedicated IOP. The secondary efficacy endpoints included the proportion of patients with Month 12 IOP of $\leq 18$ or $\leq 15 \mathrm{mmHg}$ without medications; and the proportion of patients with Month 12 IOP reduced by $\geq 20 \%$ without medication versus baseline medicated IOP. Proportional analyses were performed for the primary and secondary efficacy endpoints. For continuous variables, mean and standard deviation values were calculated. Statistical tests were performed using software from the R Project for Statistical Computing (version 3.1.3, The R Foundation, Vienna, Austria).

\section{RESULTS}

\section{Subject Accountability, Demographics and Pre-Study Parameters}

A total of 57 qualified subjects underwent $a b$ interno implantation of 2 iStent inject devices in a standalone procedure (i.e., without cataract surgery). Table 1 shows demographic and preoperative parameters. Mean age was $65.3 \pm 9.0$ years, with approximately equal gender distribution. All subjects were phakic. Preoperative mean medicated IOP was $19.5 \pm 1.5 \mathrm{mmHg}$ on 1 medication, and mean unmedicated (post-washout) IOP was $24.4 \pm 1.3 \mathrm{mmHg}$. Subjects have been followed for 18 months, and follow-up is ongoing.
Table 1 Subject demographic and preoperative characteristics $(N=57)$

\begin{tabular}{ll}
\hline Total & $\begin{array}{l}\mathbf{5 7} \text { eyes of } \mathbf{5 7} \\
\text { subjects }\end{array}$ \\
\hline Gender & \\
Male/Female & $30 / 27$ \\
Race & \\
Caucasian & $100 \%(n=57)$ \\
Age (years) & \\
Mean \pm SD & $65.3 \pm 9.0$ \\
Preoperative cup-to-disc (C:D) ratio & $0.7 \pm 0.1$ \\
mean \pm SD & \\
Preoperative medicated IOP (mmHg) & $19.5 \pm 1.5$ \\
mean \pm SD & \\
Preoperative \# medications & \\
Mean \pm SD & $1 \pm 0$ \\
Type of medications & \\
Prostaglandin & \\
Beta-blocker & \\
Carbonic anhydrase inhibitor & \\
Preoperative unmedicated IOP & \\
\hline (mmHg) mean \pm SD & $(n=49)$ \\
\hline
\end{tabular}

$I O P$ intraocular pressure, $S D$ standard deviation

\section{Efficacy}

Figure 2 shows a proportional analysis of IOP at Month 12, and Fig. 3 shows the mean IOP over time for the 18-month period after stent implantation $(N=57)$. At Month $12,100 \%$ of eyes had achieved an IOP reduction $\geq 20 \%$ (100\% had IOP $\leq 18 \mathrm{mmHg}$ and $67 \%$ had IOP $\leq 15 \mathrm{mmHg}$ ) without medication versus preoperative unmedicated IOP, and $75 \%$ had IOP reduction $\geq 20 \%$ versus preoperative medicated IOP. At Month 18, mean unmedicated IOP had decreased $41 \%$ versus preoperative unmedicated IOP (to 


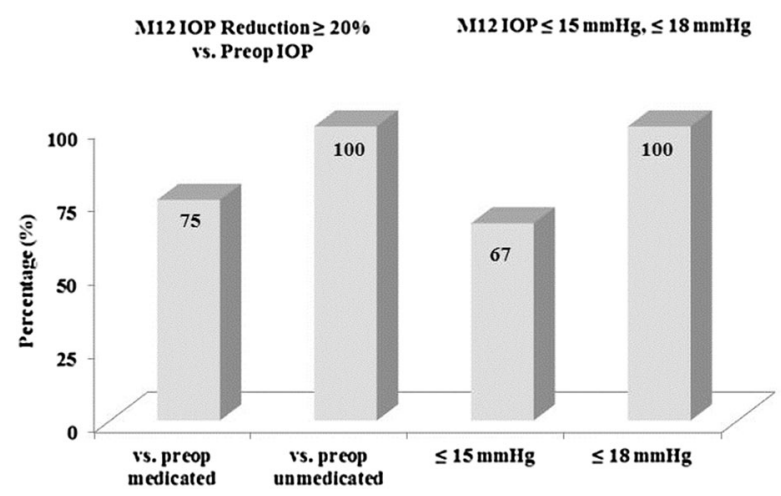

Fig. 2 Proportional analysis of Month 12 intraocular pressure (IOP) $(N=57) . M$ month

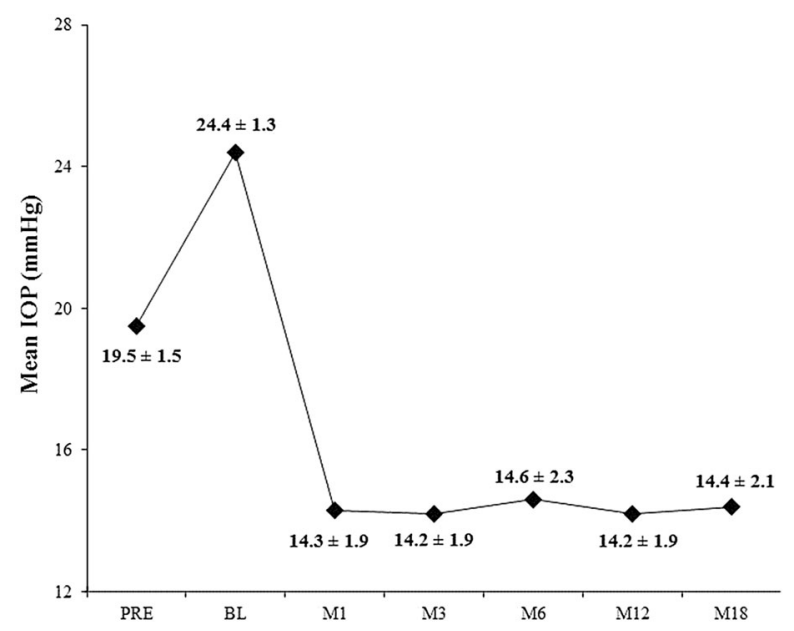

Fig. 3 Mean intraocular pressure (IOP) over time $(N=57)$. $B L$ baseline, $M$ month, PRE preoperative

$14.4 \pm 2.1 \mathrm{mmHg} \quad$ from $\quad 24.4 \pm 1.3 \mathrm{mmHg}$ preoperatively); and had decreased $27 \%$ versus preoperative medicated IOP (19.5 \pm 1.5 preoperatively). One subject was placed on a medication at Month 18 for optic nerve findings (IOP was $17.7 \mathrm{mmHg}$ ), but all remaining subjects remained off medications.

\section{Safety}

All subjects underwent uncomplicated $a b$ interno placement of 2 iStent inject devices in a standalone procedure, with no intraoperative adverse events observed. Postoperatively, 1 subject had progression of preexisting cataract with corresponding BCVA loss $>1$ line. BCVA has been stable over time for the remaining subjects (93\% with Month 18 BCVA 20/40 or better and $98 \%$ with BCVA $20 / 100$ or better) (Fig. 4). The mean C:D ratio, visual field mean deviation and pattern standard deviation, and central corneal thickness also remained stable over the 18-month follow-up (Table 2).

\section{DISCUSSION}

Data from this prospective study confirm the hypothesis that phakic subjects with OAG and on 1 ocular hypotensive medication can successfully and safely achieve sustained IOP and medication reduction following implantation of two second-generation iStent inject devices as a sole procedure. Specifically, a $\geq 20 \%$ IOP reduction with elimination of medication was achieved in all subjects at 12 months, with all patients maintaining IOP of $\leq 18 \mathrm{mmHg}$ and two-thirds reaching IOP of $\leq 15 \mathrm{mmHg}$. At Month 12, the mean unmedicated IOP had decreased by $42 \%$, to 14.2 vs $24.4 \mathrm{mmHg}$ preoperatively, and this reduction was maintained through 18 months (14.4 $\mathrm{mmHg}$ ).

These outcomes corroborate the considerable existing body of research showing substantial IOP and medication reduction and favorable safety of $a b$ interno trabecular micro-bypass implantation in patients with mild to moderate glaucoma. Prior studies have evaluated single [3-6] and multiple [7] first-generation stents (iStent) during cataract surgery; multiple first-generation stents as a standalone procedure [8-11]; and two second-generation stents (iStent inject) either with [12] or without cataract surgery in OAG 


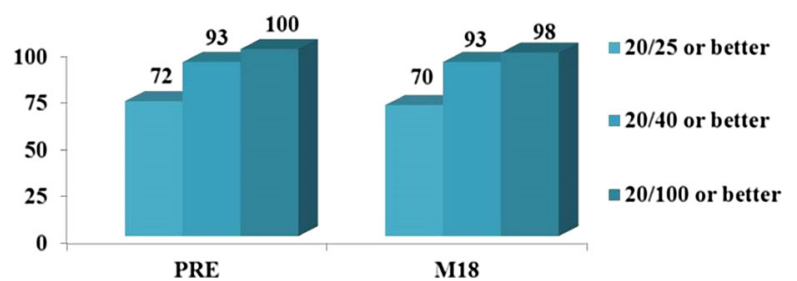

Fig. 4 Preoperative and Month 18 best-corrected visual acuity (BCVA) $(N=57)$. $M$ month, PRE preoperative

patients with moderate IOP elevation and/or medication burden [13-16]. In the present study, the observed IOP reduction of $10.2 \mathrm{mmHg}$ was similar to the $7.0-10.5 \mathrm{mmHg}$ reduction observed in these earlier studies of standalone iStent inject implantation in phakic eyes with OAG [13-16]. The present study analyzes IOP and medication use after standalone iStent inject implantation in phakic OAG eyes with IOP not managed to below $18 \mathrm{mmHg}$ on 1 preoperative medication. Thus, the findings shed light on the utility of these stents in a relatively early stage of managing the disease.

The reduction in medication-free IOP supports the independent effect of second-generation trabecular micro-bypass stents without the confounding factors of concomitant cataract surgery or medication use. Perhaps even more meaningful, subjects' postoperative IOP was $27 \%$ lower without medications 18 months after receiving 2 iStent inject devices than with medication before surgery. The elimination of medication represents a benefit in terms of both cost [19] and compliance [20-23] to glaucoma patients, most of whom have at least one other chronic condition demanding their funds and attention [24]. Indeed, an inherent advantage of $a b$ interno trabecular bypass stents over medications is reduced dependence on medication compliance, which theoretically may increase long-term treatment success.

Safety outcomes were favorable through the 18 months of follow-up, with only 1 postoperative adverse event (BCVA loss due to cataract progression; cataract surgery has been planned) and no intraoperative or device-related adverse events. Furthermore, the study's involvement of multiple surgeons offers external validation of safety of the implantation technique. This favorable safety profile is consistent with that observed in previous studies of iStent inject in subjects with mild to moderate primary OAG [12-16]. One additional medication was started in 1 eye at 18 months (IOP was $17.7 \mathrm{mmHg}$ ), but all remaining eyes remained medication-free, and no eyes underwent secondary surgery. Subject accountability was $100 \%$ through 18 months, supporting the validity of the study's findings.

Table 2 Screening and postoperative mean cup-to-disc (C:D) ratio, visual field (VF), and central corneal thickness, available eyes at each visit

\begin{tabular}{lllll}
\hline & Screening & M6 & M12 & M18 \\
\hline$N$ & 57 & 57 & 57 & 57 \\
C:D ratio mean (SD) & $0.7(0.1)$ & $0.7(0.1)$ & $0.7(0.1)$ & $0.7(0.1)$ \\
VF-mean deviation (dB) mean (SD) & $-4.9(5.3)$ & $-4.8(5.3)$ & $-4.5(5.1)$ & $-4.5(5.2)$ \\
VF-pattern standard deviation (dB) mean (SD) & $3.3(2.4)$ & $3.1(2.3)$ & $3.1(2.3)$ & $3.0(2.3)$ \\
Corneal thickness $(\mu \mathrm{m})$ mean $(\mathrm{SD})$ & $544.9(30.3)$ & $546.1(30.0)$ & $545.6(29.9)$ & $545.4(29.7)$ \\
\hline
\end{tabular}

$M$ month, $S D$ standard deviation 
This is a nonrandomized, open-label, single-arm study with several limitations which may be noted. The study's single-site design prevents a consideration of site-specific effects or measurement variability as potential confounders. Since multiple IOP readings were not taken, regression to the mean is a possibility. Because stent implantation was completed as a standalone procedure in this study, a sham surgical control group (consisting of merely injecting and removing viscoelastic) was not considered for ethical reasons. However, we considered it reasonable that patients' preoperative data served as their own control. The patient population was 100\% Caucasian and included only phakic eyes, making it impossible to stratify by race or lens status. IOP measurements at multiple time points were not taken for every visit, diurnal IOP was not measured, and standardized preoperative grading of the crystalline lens was not completed.

\section{CONCLUSIONS}

This study collected valuable data in a stable cohort with excellent accountability through 18 months, and follow-up is ongoing. The results confirm that IOP reduction to $\leq 15 \mathrm{mmHg}$ with elimination of medicationand a favorable safety profile-is possible after the standalone implantation of two iStent inject devices in OAG eyes with IOP not managed to below $18 \mathrm{mmHg}$ on 1 preoperative medication. In light of this positive benefit-to-risk profile, as well as the well-known issues with compliance, cost, and side effects associated with topical medications, this microsurgical technique may constitute a desirable alternative for these patients with earlier stages of OAG.

\section{ACKNOWLEDGMENTS}

The sponsor, Glaukos Corporation, Laguna Hills, CA, provided study devices, sponsorship for performing this study, data collection, data management, data analysis, and article processing charges. All named authors meet the International Committee of Medical Journal Editors (ICMJE) criteria for authorship for this manuscript, take responsibility for the integrity of the work as a whole, and have given final approval to the version to be published. All authors had full access to all of the data in this study and take complete responsibility for the integrity of the data and accuracy of the data analysis.

Disclosures. R. Lindstrom is a consultant and stock holder of Glaukos. R. Lewis is a consultant for Glaukos. S. Sarkisian is a consultant for Glaukos and received financial support from Glaukos for his work as an investigator in this study. L. Voskanyan received financial support from Glaukos for her work as an investigator in this study. J. Hovanesian received financial support from Glaukos for his work as an investigator in this study. J. E. Giamporcaro is an employee of Glaukos. D. Hornbeak is an employee of Glaukos.

Compliance with Ethics Guidelines. All procedures followed were in accordance with the ethical standards of the responsible committee on human experimentation (institutional and national) and with the Helsinki Declaration of 1964, as revised in 2013. Informed consent was obtained from all patients for being included in the study. 
Open Access. This article is distributed under the terms of the Creative Commons Attribution-NonCommercial 4.0 International License (http://creativecommons.org/licenses/ by-nc/4.0/), which permits any noncommercial use, distribution, and reproduction in any medium, provided you give appropriate credit to the original author(s) and the source, provide a link to the Creative Commons license, and indicate if changes were made.

\section{REFERENCES}

1. Tham Y-C, Li X, Wong TY, Quigley HA, Aung T, Cheng CY. Global prevalence of glaucoma and projections of glaucoma burden through 2040: a systematic review and metaanalysis. Ophthalmology. 2014;121(11):2081-90.

2. Rulli E, Biagioli E, Riva I, et al. Efficacy and safety of trabeculectomy versus nonpenetrating surgical procedures: a systematic review and meta-analysis. JAMA Ophthalmol. 2013;131(12):1573-82.

3. Samuelson TW, Katz LJ, Wells JM, Duh Y-J, Giamporcaro JE. Randomized evaluation of the trabecular micro-bypass stent with phacoemulsification in patients with glaucoma and cataract. Ophthalmology. 2011;118:459-67.

4. Craven ER, Katz LJ, Wells JM, Giamporcaro JE. Cataract surgery with trabecular micro-bypass stent implantation in patients with mild-to-moderate open-angle glaucoma and cataract: two-year follow-up. J Cataract Refract Surg. 2012;38:1339-45.

5. Fea AM. Phacoemulsification versus phacoemulsification with micro-bypass stent implantation in primary open-angle glaucoma. J Cataract Refract Surg. 2010;36:407-12.

6. Arriola-Villalobos P, Martinez-de-la-Casa J, Diaz-Valle D, et al. Combined iStent trabecular micro-bypass stent implantation and phacoemulsification for coexistent open-angle glaucoma and cataract: a long-term study. $\mathrm{Br} \mathrm{J}$ Ophthalmol. 2012;96:645-9.

7. Belovay GW, Naqi A, Chan BJ, Rateb M, Ahmed II. Using multiple trabecular micro-bypass stents in cataract patients to treat open-angle glaucoma. J Cataract Refract Surg. 2012;38(11):1911-7.
8. Donnenfeld ED, Solomon KD, Voskanyan L, et al. A prospective 3-year follow-up trial of implantation of two trabecular microbypass stents in open-angle glaucoma. Clin Ophthalmol. 2015;9:2057-65.

9. Katz LJ, Erb C, Carceller Guillamet AC, et al. Prospective, randomized study of one, two, or three trabecular bypass stents in open-angle glaucoma subjects on topical hypotensive medication. Clin Ophthalmol. 2015;9:2313-20.

10. Ahmed II, Katz LJ, Chang DF, et al. Prospective evaluation of microinvasive glaucoma surgery with trabecular microbypass stents and prostaglandin in open-angle glaucoma. J Cataract Refract Surg. 2014;40:1295-300.

11. Chang D. Long term findings following micro-invasive glaucoma surgery (MIGS) with two trabecular micro-bypass stents and travoprost in OAG not controlled on two preoperative medications. Presented at European Society of Cataract and Refractive Surgeons, February 2016, Athens, Greece.

12. Arriola-Villalobos P, Martinez-de-la-Casa J, Fernandez-Perez J, et al. Mid-term evaluation of the new Glaukos iStent with phacoemulsification in coexistent open-angle glaucoma or ocular hypertension and cataract. $\mathrm{Br} \mathrm{J}$ Ophthalmol. 2013;97:1250-5.

13. Fea AM, Belda JI, Rekas M, et al. Prospective unmasked randomized evaluation of the iStent inject $^{\circledR}$ versus two ocular hypotensive agents in patients with primary open-angle glaucoma. Clin Ophthalmol. 2014;8:875-82.

14. Klamann MKJ, Gonnermann J, Pahlitzsch M, et al. iStent inject in phakic open angle glaucoma. Graefes Arch Clin Exp Ophthalmol. 2015;253:941-7.

15. Voskanyan L, Garcia-Feijoo J, Belda J, et al. Prospective, unmasked evaluation of the iStent inject system for open-angle glaucoma: synergy trial. Adv Ther. 2014;31:189-201.

16. Hengerer FH, Conrad-Hengerer I. Personal experience with second generation trabecular micro-bypass stents in open angle glaucoma: 18 months follow-up. Presented at European Glaucoma Society, June 2016, Prague, Czech Republic.

17. Höh H, Grisanti S, Grisanti S, Rau M, Ianchulev S. Two-year clinical experience with the CyPass micro-stent: safety and surgical outcomes of a novel supraciliary micro-stent. Klin Monbl Augenheilkd. 2014;231(4):377-81. 
18. Sarkisian S. Combined cataract surgery and supraciliary microstent implantation for open-angle glaucoma: multicenter 3-year results. Presented at American Society of Cataract and Refractive Surgeons, May 2016, New Orleans, LA, USA.

19. Red Book ${ }^{\mathrm{TM}}$. http://micromedex.com/products/ product-suites/clinical-knowledge/redbook. Ann Arbor, Michigan: Truven Health Analytics; c2013. Accessed 6 July 2016.

20. Robin AL, Novack GD, Covert DW, et al. Adherence in glaucoma: objective measurements of once-daily and adjunctive medication use. Am J Ophthalmol. 2007;144:533-40.

21. Muir KW, Lee PP. Glaucoma medication adherence; room for improvement in both performance and measurement [editorial]. Arch Ophthalmol. 2011;129:243-5.

22. Schwartz GF, Reardon G, Mozaffari E. Persistency with latanoprost or timolol in primary open angle glaucoma suspects. Am J Ophthalmol. 2004;137:S13-6.

23. Olthoff CM, Schouten JS, van de Borne BW, Webers CA. Noncompliance with ocular hypotensive treatment in patients with glaucoma or ocular hypertension an evidence-based review. Ophthalmology. 2005;112:953-61.

24. Salim S, Shields MB. Glaucoma and systemic diseases. Surv Ophthalmol. 2010;55:64-77. 